\title{
Management of "Lens Induced Glaucoma”-A Clinical Study
}

\author{
Dr. Jyoti Bhuyan ${ }^{1}$, Dr. Mayur Dutta Bharali ${ }^{2}$. \\ ${ }^{1}$ Professor,Regional Institute Of Ophthalmology, Gauhati Medical College And Hospital, Guwahati, Assam, India \\ ${ }^{2}$ Post Graduate Trainee,Regional Institute Of Ophthalmology, Gauhati Medical College And Hospital, Guwahati, Assam, India
}

\begin{abstract}
:
Aim: To study the incidence of lens induced glaucoma (LIG), various modes of presentation in different types of LIGs and to assess the visual outcome following management of LIGs.

Materials And Methods: The present study was conducted on the patients who presented with Lens induced glaucoma in the outpatient department of the Regional Institute of Ophthalmology, Gauhati Medical College \& Hospitals, Guwahati during the period September 2014 to August, 2015. Altogether 51 patients presented with lens induced glaucoma during this period of study. All patients were admitted in the ophthalmology department. Informed and written consents were taken. All cases diagnosed as LIG irrespective of age, sex and clinical types were clinically investigated and managed.

Results: The data were compiled using a standardized performa and at the end of the study the data was analyzed statistically using the Chi-Square method and the Probability value. Incidence of LIG during the period of study was $0.15 \%$ amongst all OPD attendance. Phacomorphic glaucoma (58.86\%) was higher in incidence compared to Phacolytic glaucoma (33.33\%) followed by $5.82 \%$ of lens particle glaucoma and $3.92 \%$ of glaucoma secondary to lens dislocation. In our study $58.82 \%$ of cases achieved best-corrected visual acuity at the end of final follow up at 6 weeks. $35.29 \%$ cases achieved visual acuity between 6/18-6/60 and 5.88\% regained visual acuity less than 6/60.

Conclusion: Early diagnosis and treatment of mature cataract is very important since delayed treatment of LIG may result in poor visual outcome. There is a great need to impart health education to the public about the importance of timely surgery for better visual outcome.
\end{abstract}

Keywords: LIG, Phacomorphic glaucoma, Phacolytic glaucoma, SICS

\section{Introduction}

Glaucoma is a diverse group of eye diseases with multifactorial etiology, characterized by an acquired loss of retinal ganglion cells, progressive optic neuropathy with morphological abnormalities in the optic nerve head and visual field defects, in which raised intraocular pressure (IOP) is a major and only modifiable risk factor $^{[1]}$

The estimated prevalence of glaucoma in the world was 60.5 million in 2010 and is expected to increase to 79.6 million by 2020 .Studies have shown that approximately 12 million Indians have already been affected by the diseases by the year 2010 and with greater life expectancy and rapidly growing ageing population this figure has been estimated to increase to 16 million by $2020^{[2]}$.

Glaucoma is a lifelong disease and is a leading cause of irreversible but preventable blindness. It has been reported that glaucoma affects the quality of life to a greater degree in India than in the west ${ }^{[3]}$.

Lens induced glaucoma (LIG) is a type of secondary glaucoma and is an ophthalmic emergency. The crystalline lens may be associated with glaucoma if dislocated either due to trauma or certain inherited disorders. In many of the instances, glaucoma is found in association with cataract. In the year 1900 Gifford and Von Reuss first described LIG in relation to cataract ${ }^{[4]}$. Various conditions which lead to diversity of presentation of glaucoma include-Lens dislocation due to trauma or certain inherited disorder(EctopiaLentis), blockage of aqueous flow through pupil due to subluxation, tilt or dislocation of lens (pupillary block) and dislocation and degeneration of lens releasing materials that obstruct aqueous outflow (phacolytic glaucoma). A cataractous lens may also lead to glaucoma by obstruction of trabecular meshwork with protein and macrophages (phacolytic glaucoma), lens particles and debris (lens particle glaucoma) or inflammatory cells as a part of immune response to lens protein antigen (phacoanaphylactic glaucoma). A swollen or intumescent lens may lead to pupillary block and secondary angle closure glaucoma (phacomorphic glaucoma) ${ }^{[5,6,7]}$.

There is cataract backlog of around 12 million in India, which is annually increasing at an estimated rate of 3.8 million. This huge backlog of ever-increasing cataract is due to increased life expectancy, rapidly growing ageing population and disparity between rate of new cases and rate of surgical removal. The occurrence of Lens Induced Glaucoma in India has high probability ${ }^{[8]}$. Thus an analysis of magnitude of lens induced glaucoma and assessment of the visual outcome after cataract surgery in patients attending tertiary care centre like Regional Institute Of Ophthalmology(RIO), Guwahati, would provide important information regarding the morbidity produced by this condition and its effect on incidence of blindness.

This study was undertaken in Regional Institute Of Ophthalmology, GMCH, Guwahati for the period from September 2014-August 2015 with the aims and objectives to study the incidence of Lens Induced 
Glaucoma(LIG), the various modes of presentation in different LIG and the visual outcome following management of LIG.

\section{Materials And Methods}

The present study was conducted in the Regional Institute of Ophthalmology, Gauhati Medical College \& Hospitals, Guwahati during the period from September 2014 to August 2015. Altogether 51 patients diagnosed as lens induced glaucoma who were admitted in the ophthalmology department irrespective of age, sex, clinical types were included in this study. Informed and written consents were taken.

Inclusion criteria included clinically diagnosing the various types of LIGs such as phacomorphic glaucoma, phacolytic glaucoma, lens particle glaucoma, glaucoma secondary to ectopialentis/lens displacement glaucoma/phacotopic glaucoma and phacoantigenic glaucoma ( phacoanaphylactic glaucoma.)

Exclusion criteria included cases of primary open angle glaucoma, cases of lens induced glaucoma with previous posterior segment pathology and corneal scarring or opacity which would hamper evaluation of visual outcome.

\section{Patients evaluation :}

Detailed history in relation to the causation of glaucoma in the affected eye was ascertained. Thorough clinical examinations including Visual acuity, Tonometry, Slit lamp biomicroscopy, Gonioscopy were done. Biometry of the affected eye and the fellow eye was done whenever possible and IOL power calculation was done for both ACIOL and PCIOL. B- scan was done in the affected eye as the fundus was not visible to rule out posterior segment pathology. Best corrected visual acuity was checked and recorded at the time of presentation, after surgery, and at each follow up visit using snellen's chart/landolt's C chart/ E chart.

Other routine tests like syringing, blood pressure recording were done.

A thorough systemic examination including examination of the cardiovascular system, respiratory system, gastrointestinal system, central nervous system, was done to exclude any other systemic diseases in all the cases. Laboratory investigations include

1. Blood investigation-TLC,DLC,ESR, haemoglobin, random blood sugar, serum creatinine.

2. R/E Urine

3. Chest X-ray

4. ECG

\section{Management}

Medical management:Medical management to bring down IOP include anti glaucoma drugs like beta-blocker( Timolol meleate $0.5 \%$ ), topical carbonic anhydrase inhibitors dorzolamide in combination with tiomololmeleate, oral carbonic anhydrase inhibitors( Tablet acetazolamide $250 \mathrm{mg}$ ), hyperosmotic agents like Mannitol (20\%),glycerol. Topical steroid either Prednisolone acetate (1\%) or Dexamethasone-antibiotic combination drops were used to bring down inflammation.

Nd:YAG laser iridotomy: It was done in 3 cases of angle closure with pupillary block and/or with presence of peripheral anterior synachiae, in whom medical management failed to decrease the IOP.

\section{Surgical management:}

The preferred surgery was Manual Small Incision Cataract Surgery with PCIOL implantation. Suitable modifications in the surgical steps were made according to the situation encountered. Triple procedure- Small incision cataract surgery with PCIOL implantation with trabeculectomy was planned in patients whose IOP was not responding to the medical management and who presented late with more than 7 days of symptoms and whose gonioscopy showed more than three quarter of peripheral anterior synachiae.

In cases of subluxated lens with $<180$ degree zonular dehiscence, and in the presence of minimum capsular support, sulcus placement of IOL was done.

All patients were given topical antibiotic steroid combination drop and a short acting cycloplegic drop in the postoperative period. Systemic steroid was given where indicated. Follow up of all the patients were done on first postoperative day, at two weeks and after six weeks. Best corrected visual acuity (BCVA) was recorded and required spectacle correction was prescribed at the end of 6 weeks.

Incidence

\section{Results}

The incidence of LIG among all patients presenting to the outpatient department of RIO is shown in table 1. Table 1.Incidence of LIG 


\begin{tabular}{|l|c|}
\hline Total no. of cases attending the outpatient department of RIO & 31944 \\
\hline Total no. of Lens Induced Glaucoma & 51 \\
\hline Incidence & 0.159 \\
\hline
\end{tabular}

The incidence of lens-induced glaucoma during the period of present study was 0.159 .

Types Of Lens Induced Glaucoma

Table 2: Incidence of different types of LIG

\begin{tabular}{|l|l|l|}
\hline Types of LIG & NO. OF PATIENTS & PERCENTAGE (\%) \\
\hline PHACOMORPHIC GLAUCOMA & 29 & 56.86 \\
\hline PHACOLYTIC GLAUCOMA & 17 & 33.33 \\
\hline LENS PARTICLE GLAUCOMA & 03 & 5.88 \\
\hline $\begin{array}{l}\text { SUBLUXATION/DISLOCATION/ } \\
\text { PHACOTOPIC GLAUCOMA }\end{array}$ & 02 & 3.92 \\
\hline PHACOANAPHYLACTIC GLAUCOMA & 0 & 0 \\
\hline
\end{tabular}

Our study showed highest number of phacomorphic glaucoma(56.86\%) followed by phacolytic glaucoma $(33.33 \%)$.

Sex Distribution

Table 3: Sex distribution

P-Value $=0.0418$

\begin{tabular}{|l|l|l|}
\hline Sex & Number of cases. & Percentage \\
\hline Male & 20 & $39.22 \%$ \\
\hline Female & 31 & $60.78 \%$ \\
\hline Total & 51 & $100 \%$ \\
\hline
\end{tabular}

This study showed a higher incidence LIG in female $(60.78 \%)$ than male(39.22\%).

\section{Age Distribution}

Table 4: Age distribution

\begin{tabular}{|l|c|c|c|}
\hline Age in years & $40-55$ & $56-70$ & $>71$ \\
\hline Males & 04 & 06 & 10 \\
\hline Females & 05 & 18 & 08 \\
\hline Total & 09 & 24 & 18 \\
\hline PERCENTAGE & $17.64 \%$ & $47.05 \%$ & $35.29 \%$ \\
\hline
\end{tabular}

$\chi 2=4.514, \mathrm{p}$-value $=0.3856$

This study revealed that LIG is more prevalent in the elderly population. Among 51 cases majority 24(47.05\%) cases were in the age group of 56-70 years.

Duration Of Symptoms

Table 5: Duration of symptoms

\begin{tabular}{|l|l|l|}
\hline Duration(weeks) & No. Of patients & Percentage (\%) \\
\hline < 1 week & 26 & 50.98 \\
\hline 1-2 week & 18 & 35.29 \\
\hline $\mathbf{2 - 4}$ week & 04 & 7.84 \\
\hline$>$ 4 week & 03 & 5.88 \\
\hline
\end{tabular}

$50.98 \%$ of the cases presented within 7 days of developing symptoms and the rest $49 \%$ presented beyond 7 days.

\section{Iop At Presentation}

Table 6: IOP at presentation

\begin{tabular}{|l|l|l|}
\hline IOP(mm of hg) & NO. OF PATIENTS & PERCENTAGE $(\%)$ \\
\hline $20-30$ & 05 & 9.80 \\
\hline $31-40$ & 15 & 29.41 \\
\hline $41-50$ & 25 & 49.02 \\
\hline$>50$ & 06 & 11.76 \\
\hline
\end{tabular}

Majority of cases 25 had IOP more than $40 \mathrm{~mm}$ of $\mathrm{Hg}$ at presentationand 6 of them had IOP more than 50 $\mathrm{mm}$ of $\mathrm{Hg}$.

\section{Visual Acuity Of The Affected Eye At Presentation:}


Table 7: Visual acuity at presentation

FC-finger counting

\begin{tabular}{|l|c|}
\hline Visual acuity & No. of patients \\
\hline FC at $1 \mathrm{~m}$ & 06 \\
\hline HMCF & 24 \\
\hline PL+ & 19 \\
\hline PL/PR doubtful & 02 \\
\hline
\end{tabular}

HMCF-hand movement close to face

PL- perception of light

PR- projection of rays

Out of 51 cases maximum number of cases(24) had hand movement followed by P/L + in 19 cases, F/C at $1 \mathrm{~m}$ in 6 cases and PL/PR doubtful in only 2 cases.

Management And Its Responses:

Table 8:Surgical procedure

\begin{tabular}{|l|l|l|l|l|}
\hline Nature of Surgery & Phacomorphic & Phacolytic & Lens particle & $\begin{array}{l}\text { Lens } \\
\text { subluxation }\end{array}$ \\
\hline Combined surgery & $\mathbf{1 4}$ & - & - & - \\
\hline SICS with PC IOL & $\mathbf{1 2}$ & $\mathbf{1 7}$ & $\mathbf{3}$ & $\begin{array}{l}\text { 1(lens extraction) } \\
\text { (PCIOL in sulcus) }\end{array}$ \\
\hline $\begin{array}{l}\text { SICS PC IOL with } \\
\text { Iridectomy }\end{array}$ & $\mathbf{0 3}$ & - & - & - \\
\hline Total & $\mathbf{2 9}$ & $\mathbf{1 7}$ & $\mathbf{3}$ & $\mathbf{2}$ \\
\hline
\end{tabular}

\section{Iop Distribution :}

Table 9: IOP distribution during immediate post-operative period

\begin{tabular}{|l|l|l|l|l|l|}
\hline Post-operative IOP & Phacomorphic & Phacolytic & Lens Particle & $\begin{array}{l}\text { Dislocated } \\
\text { /subluxated }\end{array}$ & Total \\
\hline$<\mathbf{2 0} \mathbf{~ m m ~ o f ~ H g ~}$ & $24(82.76 \%)$ & $15(88.24 \%)$ & $3(100 \%)$ & $2(100 \%)$ & $44(86.27 \%)$ \\
\hline$>\mathbf{2 0} \mathbf{~ m m}$ of Hg & $5(17.24 \%)$ & $2(11.76 \%)$ & 0 & 0 & $7(13.73 \%)$ \\
\hline Total & $29(100 \%)$ & $17(100 \%)$ & 3 & 2 & $51(100 \%)$ \\
\hline
\end{tabular}

Majority $(86.27 \%)$ cases in our study had reduction in intraocular pressure after surgery to less than 20 $\mathrm{mm}$ of $\mathrm{Hg}$. And only $7 \%$ had IOP more than $20 \mathrm{~mm}$ of $\mathrm{Hg}$ after surgery. IOP control was seen more in phacolytic cases $(88.24 \%)$ in our series. After cataract extraction, IOP could be controlled without the need of anti-glaucoma drugsin 44(86.27\%)cases out of 51 cases.In the 7 cases whose IOP was more than $20 \mathrm{~mm}$ of $\mathrm{Hg}$ was managed with steroid drop.

\section{Visual Outcome:}

Out of 51 cases under the study, 30 cases regained a visual acuity of $6 / 12$ or better (58.82\%) and 18 cases $(35.29 \%)$ achieved moderate visual acuity between 6/18-6/60. Rest of the cases $(5.88 \%)$ had a final best corrected visual acuity( BCVA) worse than 6/60.

Table 10: Final BCVA and Age

\begin{tabular}{|l|l|l|l|}
\hline Age & $\begin{array}{c}\text { 6/6-6/12 } \\
\text { Good vision } \\
\text { (no. of eyes/ percentage) }\end{array}$ & $\begin{array}{l}>6 / 18 \text { Poor vision } \\
\text { (no. of eyes/ percentage) }\end{array}$ & Total \\
\hline$<50$ years & $\mathbf{0 4}(66.66 \%)$ & $02(33.33 \%)$ & 06 \\
\hline$>50$ years & $26(57.78 \%)$ & $19(42.22 \%)$ & 45 \\
\hline Total & 30 & 21 & 51 \\
\hline
\end{tabular}

\section{$P$ value-1.000}

In this series 66.66 patient and BCVA is not statistically significant.\% of caseswho were less than 50 years achieved good visual acuity in comparison to $57.78 \%$ who were above 50 years. In our study age of the patient and BCVA after surgery was not statistically significant.

Table 11: Final BCVA and duration of symptoms

\begin{tabular}{|l|l|l|}
\hline DURATION & $6 / 6-6 / 12$ I & $>6 / 18$ \\
\hline$<7$ days & $\mathbf{2 0}(\mathbf{7 4 . 0 7 \%})$ & $\mathbf{0 7}(\mathbf{2 5 . 9 2})$ \\
\hline$>7$ days & $\mathbf{1 0}(\mathbf{4 1 . 6 7 \%})$ & $\mathbf{1 4}(\mathbf{5 8 . 3 3})$ \\
\hline Total & $\mathbf{3 0}$ & $\mathbf{2 1}$ \\
\hline P value $=0.249$ & & \\
\hline
\end{tabular}


In our study we observed that 20 cases(74.07\%) who presented early (less than 7 days) achieved good visual acuity. On the other hand only 10 cases (41.67\%) who presented late (more than 7 days) achieved good vision. The association between duration of symptoms and best corrected visual acuity was statistically significant in this study, $\mathrm{p}$ value $=0.0249$.

Table 12: BCVA and IOP at presentation.

\begin{tabular}{|l|l|l|l|}
\hline IOP & $\begin{array}{c}\text { 6/6-6/12 } \\
\text { Good vision }\end{array}$ & $\begin{array}{l}>6 / 18 \\
\text { Poor vision }\end{array}$ & Total \\
\hline $\begin{array}{l}\text { S40 mm of } \\
\text { Hg }\end{array}$ & $13(65 \%)$ & $7(35 \%)$ & $20(100 \%)$ \\
\hline$>40 \mathrm{~mm}$ of Hg & $17(54.84 \%)$ & $14(45.16 \%)$ & $31(100 \%)$ \\
\hline Total & 30 & 21 & 51 \\
\hline
\end{tabular}

\section{$P$ value- 0.5659}

In our study association between pre-operative IOP and BCVA was not statistically significant where $65 \%$ and $54.84 \%$ cases who had IOP less than $40 \mathrm{~mm} \mathrm{Hg}$ and more than $40 \mathrm{~mm}$ of $\mathrm{Hg}$ respectively at presentation regained good vision.

\section{Discussion}

The present study was conducted on 51 cases of LIG .The patients were followed up from the time of admission to surgery and beyond 6 weeks post-operatively and the factors influencing the final visual outcome were analysed. Statistically Chi-Square test $(\chi 2)$ and Probability values $(\mathrm{p})$ were used and $\mathrm{p}$-value $<0.05$ was considered statistically significant.

\section{Incidence:}

Incidence of LIG in this studywas $\mathbf{0 . 1 5 9 \% [ T a b l e ~ n o . 1 ] ~ o f ~ t h e ~ t o t a l ~ c a s e s ~ a t t e n d i n g ~ o u t p a t i e n t ~ d e p a r t m e n t . ~}$ The incidence of LIG in this study was marginally less but comparable to the observation by SaikiaA.K ${ }^{[9]}$ ,and much lower than that of previous report by Dutta et $\mathrm{al}^{[10]}$. This relatively lower incidence is probably due to the effective implementation of the National Programme for Control of Blindness under which multiple eye camps are being conducted round the year in the catchment area of the Regional Institute of Ophthalmology.

\section{Types Of LIG}

In our study it was observed that the most frequent type of LIG was Phacomorphic glaucoma $(58.86 \%)$ followed by Phacolytic glaucoma (33.33\%)[Table 2].Similar occurrence was noted by different authors.

Percentage of different types of LIG by different Authors.

\begin{tabular}{|l|l|l|}
\hline & Phacomorphic & Phacolytic \\
\hline Prajna et. al. (1996)[11] & $52.7 \%$ & $47.3 \%$ \\
\hline Pradhan et. al. (2001)[12] & $72 \%$ & $28 \%$ \\
\hline Rijal AP (2006)[13] & $65 \%$ & $35 \%$ \\
\hline Murty et. al. (2015)[14] & $62 \%$ & $38 \%$ \\
\hline Our study & $58.86 \%$ & $33.33 \%$ \\
\hline
\end{tabular}

\section{Sex Distribution:}

In this study, females seemed to have an increased risk of having LIG compared to males with ratio of 1.6:1. It was observed that the majority (60.78\%) of cases occurred among females and $39.22 \%$ cases occurred in males. In this study $\mathrm{p}=0.04$ showed that LIG being common in female was statistically significant. [Table-3]

Similar results were reported by SaikiaAK ${ }^{[9],} \operatorname{RijalAP}^{[13]}$, Pradhan et al. ${ }^{[12]}$.They observed that socio-economic and cultural constraints play a role leading to neglect and late presentation of cataract in females in region where the study was done. In our study we also found that these entities are more common in females because of socio - economic constraints.

\section{Age Distribution:}

Our study observed that LIG was more common in elderly people, particularly in the fifth decade and above. Highest number of cases occurred in the age group 56-70 years (47.05\%)[Table-4]. In our study $\chi^{2}=4.514$ and $\mathrm{p}$-value $=0.385$ showing that no significant association existed between the age group of 
patients and the disease in this study. Similar observations were made by Pradhan et $\mathrm{al}^{[12]}$, Saikia $\mathrm{AK}^{[9]}$, Dhar et al ${ }^{[15]}$.

Occurrence of LIG in the older age group is attributed to the fact that cataract is neglected till they become hypermature and producing symptoms other than dimness of vision and pain requiring immediate medical help. This is because of insidious onset, lack of medical awareness, lack of regular eye check-up, ignorance and limited resourses in developing countries.

\section{Duration Of Symptoms:}

Majority of cases 26(50.98\%) presented within the first week of developing symptoms and rest 25 cases $(49.01 \%)$ presented after 1 week in our study. Pradhan et $\mathrm{al}^{[12]}$ in his study found $70 \%$ of cases presented after 10 days of onset of symptoms. He stated the reasons for late presentation were "no escort" and "lack of money". We observed similar findings in our study. Most of the patients who presented with LIG were from rural and semi urban areas and of poor socioeconomic background. It was observed that literacy among the cases was low and many of them were from far flung areas without any nearby facility of eye care service. This might be one of the causes for late presentation in our study.

\section{IOP At Presentation:}

In our series $25(49.02 \%)$ cases presented with IOP of more than $40 \mathrm{~mm}$ of $\mathrm{Hg}$.and 6 cases $(11.76 \%)$ had IOP more than $50 \mathrm{~mm}$ of $\mathrm{Hg}$ [Table 6].Our results were similar to the study done by Dhar et $\mathrm{al}^{[15]}$.

\section{Surgical management:}

In our study out of 29 cases of phacomorphic glaucoma, Combined surgery (SICS+PCIOL+Trabeculectomy) was done in 14 cases. Combined surgery was done in cases with longer duration of attack. SICS with PC IOL was done for remainder of cases of phacomorphic glaucoma cases, with short duration of attack. SICS with PC IOL with iridotomy done, in three case of phacomorphic glaucoma[Table-8]. Laser iridotomy was done in three of the cases of phacomorphic glaucoma preoperatively, where IOP was not controlled with maximum medical management.

There were 17 Phacolytic glaucoma cases. SICS with PC IOL was done in all those cases. Out of the two cases of lens dislocation/subluxation, one case was managed by lens extraction only due to complete zonular dehiscence and in one case PCIOL was placed in the sulcus. All the three cases of lens particle glaucoma were surgically managed with small incision cataract surgery with posterior chamber IOL implantation[Table-8].

In our series we managed to do sutureless cataract surgery in all the cases. Pre-operative IOP control in all those cases were achieved medically to acceptable limits before going for definitive surgery.

\section{Visual Outcome:}

Best corrected visual acuity (BCVA) and its association with Age, IOP and duration of symptoms at presentation. BCVA of $6 / 12$ or better was considered as good visual acuity and 6/18 or less as poor vision.

\section{Association between final BCVA and Age}

In this study [Table-10], 4cases of age less than 50 years and 26 cases of age more than 50 years achieved good visual acuity of better than $6 / 12$. We observed that there was no statistically significant association between age at presentation and BCVA ( $\mathrm{p}$ value $=1.000$ ). Our study is comparable to Prajna et $\mathrm{al}^{[11]}$ who observed significant risk of poor visual outcome in cases of age higher than 60 years when compared with younger patients.

Our study showed that 21 cases had best corrected visual acuity of $6 / 18$ or less [Table-10]. The causes of poor visual acuity outcome was attributed to late presentation leading to glaucomatous damage to the optic nerve head and ARMD.

\section{Association between BCVA and duration of symptoms}

In this study[Table-11], 52.64\% cases presented earlier than 7 days and $74.07 \%$ regained good visual outcome. Whereas among the $47.05 \%$ cases who presented beyond 7 days only $41.66 \%$ cases achieved good visual acuity of $6 / 12$ or better. It showed that a significant risk of poor visual acuity was found when the duration between the onset of symptom and presentation to our OPD was more than 7 days $(\mathrm{p}$ value $=0.0249)$. Similar observation was made by Prajna et $\mathrm{a}^{[11]}$ where a significant risk of poor visual acuity was found when the duration between the onset of pain and surgery exceeded five day. 


\section{Association between BCVA and IOP}

In our study[Table-12] 13 cases $(65 \%$ ) with IOP at presentation less than $40 \mathrm{mmHg}$ achieved good visual acuity, whereas cases with IOP more than $40 \mathrm{~mm} \mathrm{Hg}(54.83 \%)$ also had good visual outcome. The correlation between pre-operative IOP and visual outcome was, clinically significant but statistically not significant $(\mathrm{p}$ value $=0.5659)$. Madurai study (Prajna et al ${ }^{[11]}$ also had found no statistically significant association between the level of preoperative IOP and final visual acuity. ( $p$ value $=0.07$ )

\section{Conclusion}

LIG is an important vision-threatening disease and it comprises a number of different glaucomatous processes occurring in the elderly that share in common, the role of the crystalline lens in the mechanism of increase in IOP. Reduced vision, eye pain and redness are the main clinical presentations of LIG. Despite high IOP at the initial presentation in cases of lens induced glaucoma, IOP came down to normal limits after lens extraction. Early diagnosis and treatment of mature cataract is very important since delayed treatment of lens-induced glaucoma may result in poor visual outcome. There is a great need to impart health education to the public about the importance of timely surgery for better visual outcome and the dangers of poor visual result if cataract surgery is delayed. The role of community outreach programs in early diagnosis of cataract cannot be undermined because success of such initiatives will lead to decrease in the incidence of Lens Induced Glaucoma in the community.

\section{Bibliography}

[1]. DadaT,IchhpujanP,LingamV,RamaswamiK,KaushikS,VyasP,SarmaP.Guidelines for medical management of primaryopen angle glaucoma.All India ophthalmological society.2011;p6

[2]. QuingleyHA,BromanAT.The number of people with glaucoma worldwide in 2010 and 2020,Br J Ophthalmology 2006;90(3):262-267

[3]. Shields textbook of glaucoma, $6^{\text {th }}$ edition

[4]. Dr. R. Jose.community health J 2008;21(65)s103-S104.

[5]. VijayaL.GoorgeR, ArvindH,BaskaranM,RajuP,RamesauseshSV,etal.Prevalance and causes of blindness in the rural population of Chennai Glaucoma study.

[6]. www.npcb.nic.in,Nationalprogramme for control of blindness(NPCB), Director General of Health and Family Welfare, Govt .of India.

[7]. Dr. Manish pande,Dr. Ronnie Jacob George, Dr.LingamVijaya. Chapter Glaucoma in India .Glaucoma Diagnosis and Management Pathways.page:3

[8]. GuptaV, SrinivasanG,Meiss,GazzardG, SihotaR, KapoorKS. Utility values among glaucoma patients: An impact on qualityof life Br.J ophthalmology 2005;89:1241-4.

[9]. Saikia AK: A clinical study on lens induced glaucoma: A thesis submitted to Gauhati University in 1992

[10]. Dutta L,Baruah CK, SarmaBK.Paper submitted to congress of Asia Pacific Academy of Ophthalmology, 1985. New Delhi

[11]. Prajna N V, Ramakrishnan R, Krishnadas R, Manoharan N. Lens induced glaucomas - visual results and risk factors for final visual acuity. Indian J Ophthalmol 1996; 44:149-55

[12]. Pradhan D, Hennig A, Kumar J, Foster A., A prospective study of 413 cases of lens-induced glaucoma in Nepal. Indian J Ophthalmol. 2001 Jun;49(2):103-7.

[13]. Rijal AP, Karki DB. Visual outcome and IOP control after cataract surgery in lens inducedglaucomas. KathmanduUniversity Medical Journal (2006), Vol. 4, No. 1, Issue 13, 30-33.

[14]. Murty et al. A clinicalStudyogf Lens Induced Glaucoma. International Journal of Scientfic research and management(IJSRM).2015

[15]. Dhar G L, Bagotra S, Bhalla A. Lens induces glaucoma-a clinical study. Indian J Ophthalmol 1984; 32:456-9

[16]. DondonaL,DandonaR, JohnRK; Natl Med J,2001, Nov-Dec; 14(6):327-34. 